\title{
Improved constraints on the cosmological parameters using the VLA FIRST survey
}

\author{
E. Xanthopoulos ${ }^{*, \dagger}$, R. H. Becker ${ }^{*, \dagger}$, W. H. deVries ${ }^{*, \dagger}$ and R. L. White ${ }^{* *}$ \\ *University of California Davis, Department of Physics, Davis, CA 95616 \\ ${ }^{\dagger}$ IGPP/Lawrence Livermore National Laboratory, Livermore, CA 94550 \\ ${ }^{* *}$ Space Telescope Science Institute, 3700 San Martin Drive, Baltimore, MD 21218
}

\begin{abstract}
Using the final version of the VLA FIRST survey (Becker et al. 2003), the optical Sloan Digital Sky Survey Data Release 3 (DR3) quasar list and a series of carefully selected criteria, we have defined the largest homogeneous population of double-lobed sources. With the precise sample definition, the increased depth and sensitivity of the survey data, the large size of the dataset, and our self-consistent method of analysis, which addresses many of the problems associated with previous work in the area, we are able to: a) explore the correlations between the intrinsic properties of the double-lobed radio sources (the results are also confirmed by a non-parametric analysis) and study their evolution, b) place more interesting and tighter constraints on the cosmological parameters, distinguish among the different cosmology models, and determine the impact of the angular sizeredshift studies in cosmology, c) further our understanding of the behavior of the intergalactic medium (IGM) density as a function of redshift and shed more light to the quasar-radio galaxy unification issue.
\end{abstract}

Keywords: Cosmological parameters, Radio galaxies

PACS: $98.80 .-\mathrm{k}$, 98.80.Bp, 98.80.Es, 98.54.Gr

\section{INTRODUCTION}

The angular size-redshift (theta-z) relation for a cosmological population of standard rods is a powerful probe of the large-scale geometry of the Universe. However, previous attempts to measure the cosmological parameters from the angular size-redshift relation of double radio-lobed radio sources have been marred by a variety of selection effects, destroying the integrity of the data sets, and by inconsistencies in the analysis, undermining the results and leading to data consistent with a static Euclidean Universe rather than with standard Friedmann models (e.g. Wardle \& Miley 1974, Barthel \& Miley 1988, Nilsson et al. 1993). Interpretation of this observation has caused disagreements among various authors.

\section{THE SAMPLE AND SELECTION CRITERIA}

The VLA FIRST survey (Becker et al. 1995) has mapped $\sim 9000 \mathrm{deg}^{2}$ of the sky at 1.4 $\mathrm{GHz}$ to a sensitivity of $\sim 1$ mJy with 5." 4 FWHM Gaussian beam and has cataloged 111,115 sources with subarcsecond positional accuracy. We cross-correlated this survey with a sample of 51,516 quasars compiled from the optical Sloan Digital Sky Survey Data Release 3 (DR3) quasar list and the list of quasars from the $2 \mathrm{dF}$ survey. By using a lower detection limit of $3 \sigma$ for the Poisson probability distribution of finding 


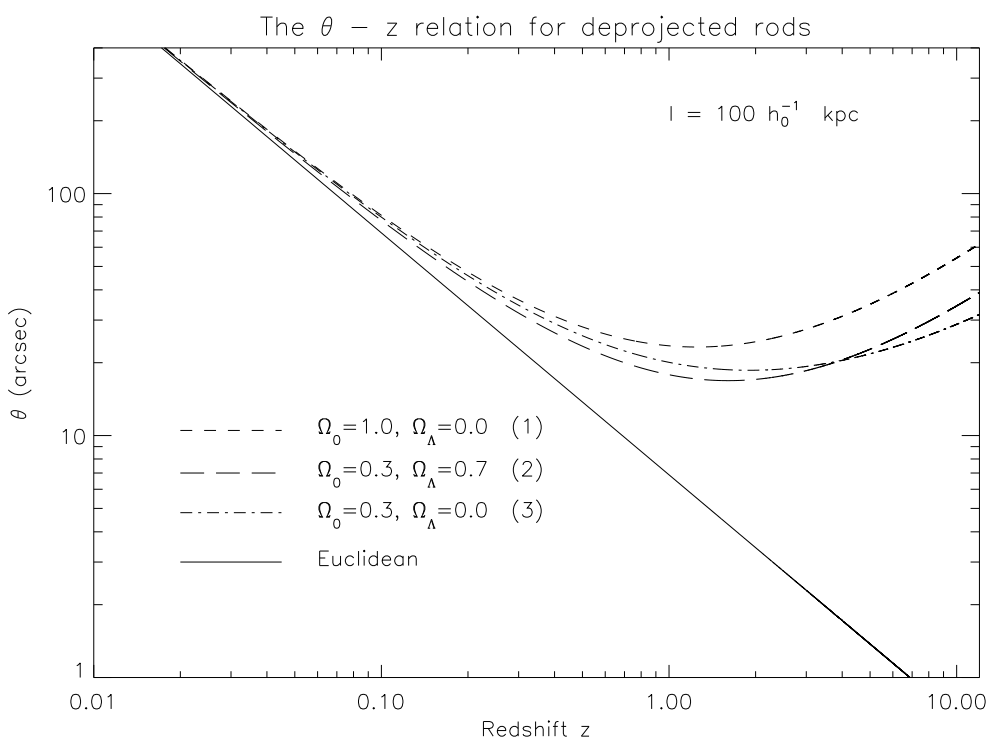

FIGURE 1. The angular size - redshift relation for deprojected rods of length $100 \mathrm{~h}_{0}^{-1} \mathrm{kpc}$ for different cosmologies. The choice of the cosmological parameters for the three Friedmann models are listed on the figure. The curve for a static Euclidean universe is shown for comparison. In practice, the curves actually define upper limits to the observed angular sizes, since projection effects will scatter the observed sizes downward. Note the presence of the minimum near $\mathrm{z} \sim 1.5$.

companions of the quasars within an area of $5^{\prime}$ radius from the quasar position, we defined an initial sample of double-lobed radio sources with their cores centered on the quasar positions. We then followed a series of carefully selected criteria, and specifically we: a) included only radio sources, FRIIs, with symmetric and collinear triple structure (core + two lobes), thereby minimizing asymmetrical effects that might distort the apparent angular size, such as relative motion with respect to the IGM, b) further restricted the sample to sources with redshift $\mathrm{z}>0.3$ beyond which quasars begin to dominate and carry more info about the cosmology. This way we bypass the problem of different mean orientations or power-size correlations which produce non- cosmological effects in the theta-z plane. Our final clean sample consists of 389 FRIIs, which is the largest homogeneous population of double-lobed sources to date.

\section{THE ANGULAR SIZE - REDSHIFT RELATIONSHIP}

Fig. 1 illustrates the $\theta-z$ relationship for deprojected rods $\left(\phi=90^{\circ}\right)$ with an intrinsic size of $\mathrm{l}=100 \mathrm{~h}_{0}^{-1} \mathrm{kpc}$ for three Friedmann cosmologies: a) an Einstein-de Sitter universe, b) a flat universe, and 3) a nonclosed, matter-dominated universe. The particular values of $\Omega_{0}$ and $\Omega_{\Lambda}$ are listed in the figure. The curve for a static Euclidean universe, with $\theta \propto z^{-1}$ is also shown for comparison. The location of the minimum in the angular size (typically between $\mathrm{z}=1$ and $\mathrm{z}=2$ ) depends on $\Omega_{0}$ and $\Omega_{\Lambda}$.

Fig. 2 shows a scatter plot of the $\theta-z$ data. The errors in the measured values of $\theta$ are typically $\sim 1^{\prime \prime}$, far less that the scatter in the angular sizes at any redshift. With 5."4 
FWHM beam, the FIRST survey can detect extended structure down to 2" . However, due to the survey resolution limit, uncertainties in the quasar optical positions, variations in the morphologies of double-lobed sources, and inspection of numerous FIRST radio maps, we also introduce an effective cutoff in the data of $12^{\prime \prime}$, shown in Fig. 2, following Buchalter et al. (1998), below which an accurate morphological classification could not be assigned with certainty. Such a cutoff ensures that we do not include the so called core-jet, diffuse, cometary and other type of extended radio sources that may be mistaken for double-lobed objects at low resolution.
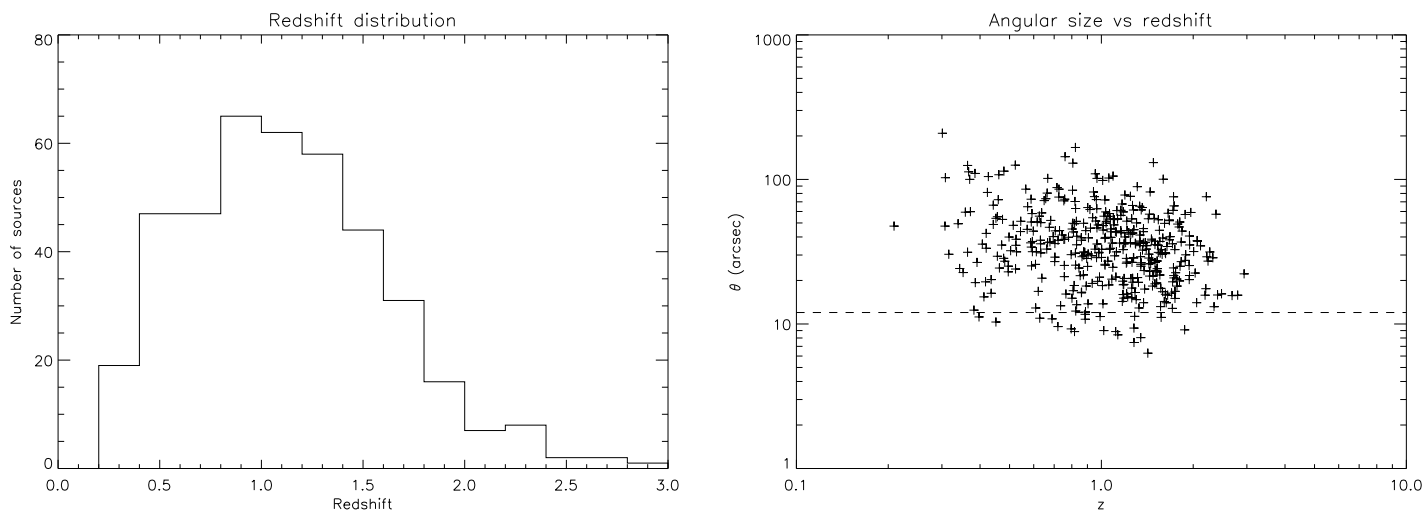

FIGURE 2. (Left:) The distribution of redshift in our sample. The sample includes sources out to a redshift of 2.94 significantly higher than the redshifts at which the minima in the theta-z curves typically occur for different Friedmann models, and in contrast to previous work which included samples that contained significant numbers of sources with $\mathrm{z}<1$ where roughly Euclidean behavior is expected. The median redshift of our sample is 1.074 while that of the FIRST survey is 1 , evidence that indicates that the two populations have similar distributions, and therefore our sample selected using the optical SDSS survey is fairly representative of double-lobed radio sources as a whole. (Right:) Scatter plot of the peakto-peak angular sizes vs redshift. The dashed line represents the effective resolution limit at $12^{\prime \prime}$, below which accurate morphological classifications could not be determined. The errors in the measured values of the angular size are typically $\sim 1^{\prime \prime}$, far less than the scatter in the angular sizes at any redshift.

\section{STANDARD AND NONSTANDARD COSMOLOGICAL MODELS}

For graphical purposes, we bin the data in redshift, in equal numbers per bin, and calculate the mean and median values of the theta, together we standard errors of the mean values and median absolute deviations for each bin. The results are shown in Fig. 3] and Fig. 4, for the median values of $\theta$, along with the curves from Fig. 1, whose amplitudes (corresponding to the median intrinsic sizes), have been scaled to provide a rough visual fit. Apart from the Friedmann models we have also experimented with the Steady State and two nonstandard cosmology models, the Tired-Light and the Gauge models, the latter shown in Fig. 4. The most striking feature of the data is that, regardless of the binning details, the observed data seem to be more consistent with Friedmann models than with a Euclidean model. The Friedmann curves shown are not the best-fit results but are merely intended for qualitative reference. 

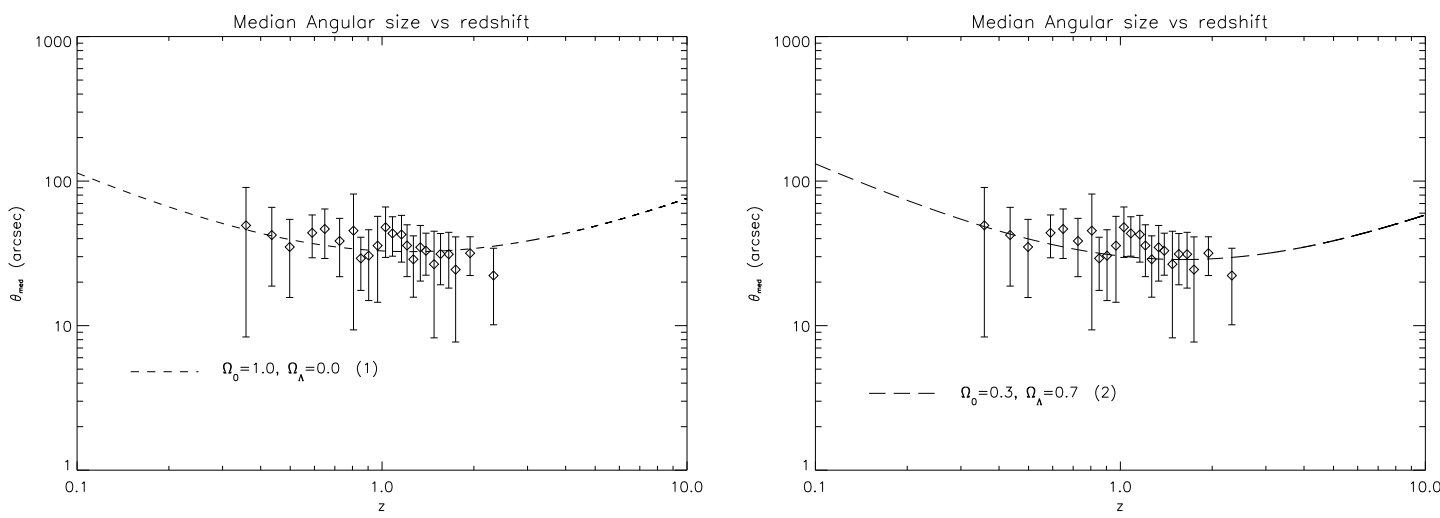

FIGURE 3. Median angular sizes, binned in redshift (to compensate for projection effects), with roughly equal numbers per bin ( $\sim$ square-root of $\mathrm{N}$ the number of sources in the sample). The Friedmann curves, corresponding to models 1 and 2 from Fig. 11 have been scaled to provide a rough visual fit. The error bars represent the median absolute deviation in each bin.
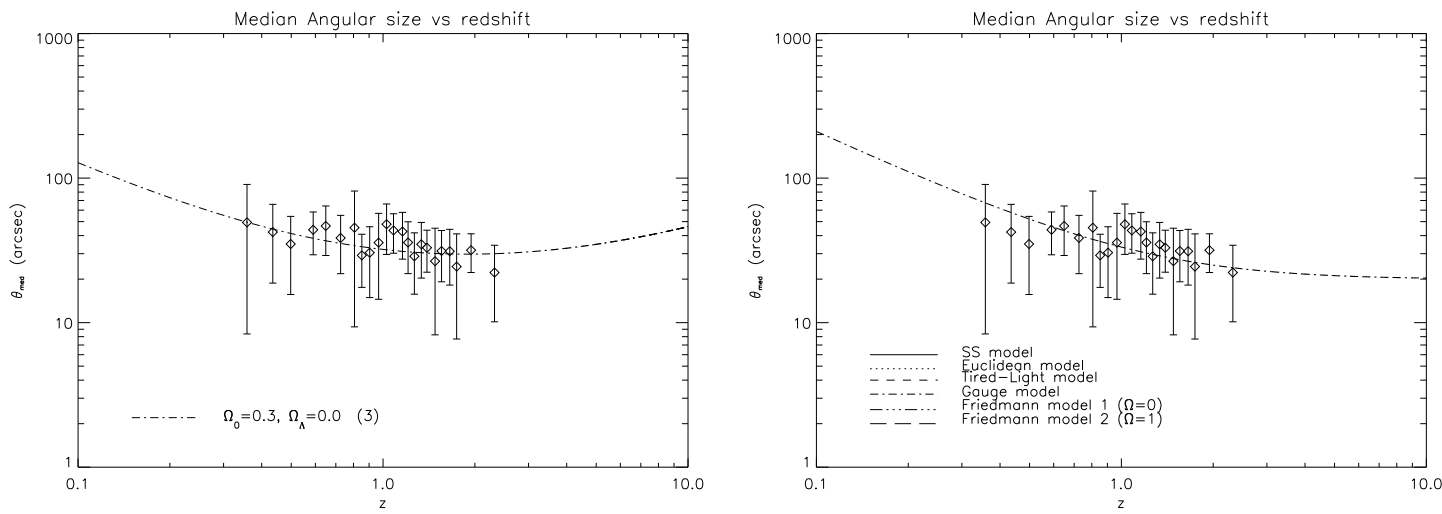

FIGURE 4. (Left:) Median angular sizes, binned in redshift (to compensate for projection effects), with roughly equal numbers per bin ( $\sim$ square-root of $\mathrm{N}$ the number of sources in the sample). The Friedmann curve, corresponding to model 3 from Fig. 11 has been scaled to provide a rough visual fit. The error bars represent the median absolute deviation in each bin. (Right:) The Gauge curve has been scaled to provide a qualitative reference. In the Gauge model, when $\mathrm{z}$ tends to infinity the angular size tends to a constant similar to the Friedmann model with qo=0 but more rapidly.

\section{DISCUSSION AND FUTURE WORK}

Before attempting to find the best-fit cosmological parameters, and determine whether we can distinguish with high significance between the different models with the present sample, we need to explore the relationships between the intrinsic properties (P intrinsic power, 1 projected linear size and redshift $\mathrm{z}$ ) of the sources in our sample using both parametric and non-parametric analysis. Such correlations have important implications in determining the parameters. Therefore our next steps are to: a) Optimize the analysis by defining and exploring a parameter space and use an iterative procedure that will 
lead to the best model fit parameters, b) Apply a chi-square goodness-to-fit statistic to determine the best fit values for the free parameters in each model and, c) Examine the values of the Hubble constant implied by the data.

\section{ACKNOWLEDGMENTS}

EXs work was performed under the auspices of the U.S. Department of Energy, National Nuclear Security Administration by the University of California, Lawrence Livermore National Laboratory under contract No. W-7405-Eng-48 and she also acknowledges support from the National Science Foundation (grant AST 00-98355).

\section{REFERENCES}

1. Barthel, P. D, Miley, G. K., Nature, 333, 319 (1988).

2. Becker, R. H., Helfand, D. J., White, R. L., Gregg, M. D., Laurent-Muehleisen, S. A., 2003, VizieR On-line Data Catalog: VIII/71.

3. Becker, R. H., White, R.L., Helfand, D. J., Astrophys. J, 450, 559-577 (1995).

4 Buchalter, A., Helfand, D. J., Becker, R. H., White, R. L., Astrophys. J, 494, 503-522 (1998).

5. Nilsson, K., Valtonen, M. J., Kotilainen, J., Jaakkola, T., Astrophys. J, 413, 453-476 (1993).

6. Wardle, J. F. C., Miley, G. K., Astron. \& Astrophy., 30, 305-315 (1974). 